\title{
Mathématiques et dialectique dans l'œuvre de Simon Stevin : l'intérêt des séries de problèmes
}

\author{
Jean-Marie Coquard ${ }^{\mathrm{a}}$ \\ ex-étudiant EHESS auprès de Giovanna Cifoletti
}

\begin{abstract}
Résumé. Dans cette contribution, je me propose de suivre quelques pistes, dans la lignée des travaux de Giovanna Cifoletti, pour approfondir les liens qui ont pu exister au XVI ${ }^{\mathrm{ème}}$ siècle entre la dialectique et l'algèbre. Ces deux disciplines avaient en effet prétention à un rôle organisateur et unificateur des savoirs. Pour cela, j'étudie les relations qu'entretiennent deux textes du même auteur, Simon Stevin (1548-1620), que je replace dans leur contexte social, historique et linguistique. On montrera ainsi que voir l'Arithmetique de ce savant comme une série de problèmes permettra d'en comprendre l'architecture, laquelle est construite d'après une méthode tirée du texte de dialectique écrit la même année (1585).
\end{abstract}

\begin{abstract}
Mathematics and dialectic in Simon Stevin's works: the interest of the notion of series of problems. The present article proposes to pursue studies, following Giovanna Cifoletti's works, about relations in the sixteenth century between dialectic and algebra. These two disciplines claimed they played a role of organization and unification of knowledge. To do so, I investigate the relationships between two treatises of the same author, Simon Stevin (1548-1620), which are considered in sociological, historical and linguistic contexts. It will be showed that looking at L'Arithmetique of Simon Stevin as a series of problems allows us to understand its architecture, which is built after a method described in the dialectic treatise written the same year (1585).
\end{abstract}

\section{Introduction}

Cette contribution se fonde sur les recherches effectuées lors de mes années de master à l'EHESS. Les objets d'études auxquels je me suis intéressé sont les liens entre les mathématiques et la dialectiquerhétorique néerlandaises du XVIème siècle, en particulier autour du savant brugeois Simon Stevin, qui a écrit sur les deux disciplines. Mon premier master a ainsi porté sur les relations entre mathématique et dialectique dans le principal texte de mathématique de Simon Stevin, L'Arithmetique. Le second avait une portée plus large et voulait expliciter les changements causés par la réforme de la dialectique de la fin du $X V^{e ̀ m e}$ siècle dans l'écriture et la pratique de différentes disciplines aux Pays-Bas : la poésie, le théâtre, la politique... Les chambres de rhétorique avaient particulièrement attiré mon attention.

\footnotetext{
a e-mail : jm-coquard@hotmail.fr
}

This is an Open Access article distributed under the terms of the Creative Commons Attribution License 4.0, which permits unrestricted use, distribution, and reproduction in any medium, provided the original work is properly cited. 
Je vais dans le présent article montrer qu'il est indispensable de connaître la dialectique de l'époque et en particulier la méthode proposée dans le texte de Stevin lui-même afin de comprendre certaines problématiques abordées par l'auteur dans L'Arithmetique : statut de la langue utilisée, place de l'algèbre par rapport à l' arithmétique, organisation des textes et des savoirs. Pour cela, je commencerai par donner quelques éléments sur l'évolution comparable de la rhétorique-dialectique et de l'arithmétique. Dans un second temps, je donnerai quelques éléments biographiques sur Simon Stevin afin de montrer en quoi étudier son œuvre est intéressant pour expliquer les convergences entre dialectique et algèbre dans le contexte des Pays-Bas de la fin du XVIème siècle. En se penchant sur la méthode décrite dans la Dialectike, nous pourrons voir que ce qui y est en jeu concerne bien plus que la rhétorique, même si les problématiques liées au langage sont fortement investies. Enfin, voir le texte de L'Arithmetique comme une série de problèmes montre que c'est une approche fructueuse pour comprendre comment une méthode dialectique est utilisée sur des savoirs mathématiques.

\section{Simon Stevin et son temps}

\subsection{Rhétorique et dialectique renaissantes}

La dialectique à la Renaissance est principalement d'inspiration cicéronienne, mêlant ainsi philosophies néoplatonicienne et stoïcienne. Elle vise essentiellement à répondre à deux questions : dans un discours, comment fait-on pour trouver les arguments et comment construit-on ce discours à partir des arguments trouvés? Ces deux questions correspondent aux deux moments de la construction du discours cicéronien : l'invention et la disposition (ou jugement). Dans le premier, on trouve les arguments, dans le second, on les met dans un certain ordre de façon à convaincre ou à persuader.

Dans l'invention, le lieu commun et l'axiome ont des statuts qui diffèrent par leur degré de certitude : le premier peut être quasiment vide de sens, il n'est qu'une banalité dont on peut se servir dans un discours (par exemple, dans une plaidoirie sur un vol, on pourra utiliser le lieu commun suivant : les pauvres veulent devenir riches), tandis que le second est un lieu commun qui a la particularité d'être considéré comme certain. Mais appliquant cette distinction aux Eléments d'Euclide, les notions communes sont les axiomes (par exemple, le tout est plus grand que sa partie) tandis que les axiomes, plus dépendants des définitions et donc plus spécifiques, sont appelés postulats. Les lieux communs et les axiomes servent alors de briques de discours qu'il faut adapter à sa situation, reprenant la valeur qu'ils avaient chez Cicéron et qui avaient été modifiée par la tradition médiévale, qui suivait plutôt Boèce.

La réforme de la dialectique de la fin du XV ${ }^{\text {ème }}$ siècle, instillée par Lorenzo Valla et le frison Rudolph Agricola ${ }^{1}$, a consisté à unifier grâce à la rhétorique les modes d'argumentation (celui en syllogismes pour les discours certains et celui en enthymèmes pour les discours ou le probable est suffisant). Elle a également eu un impact sur la forme des textes qu'elle a atteints. Dans une argumentation de type dispute médiévale, on retrouve le triptyque thèse - antithèse - synthèse. En mettant l'accent sur la recherche de la vérité par le discours argumentatif, la dialectique renaissante fait disparaitre la synthèse, qui correspondait à la vérité révélée, tandis que les thèse et antithèse ne s'opposent plus dans deux moments distincts mais sont fusionnées pour obtenir une argumentation in utramque partem selon l'expression de Cicéron, rationes contra rationes selon celle de Melanchthon, ami de Luther et réformateur de l'enseignement allemand. Là où l'on avait trois listes d'arguments (une pour la thèse, une pour l'antithèse, une pour la synthèse), les nouvelles argumentations font place à un tableau à deux colonnes où les arguments se correspondent et s'opposent un à un.

Dans les œuvres néerlandophones, ce changement se voit particulièrement bien dans les pièces de théâtre appelées Spel van Zinne (pièce de moralité) : autour des années 1530, c'est le troisième acte en

\footnotetext{
${ }^{1}$ Je m'inspire ici du livre de Peter Mack : (Mack, 2011).
} 
entier qui tombe et les personnages jouant des vices et des vertus ne se retrouvent plus les uns dans le premier acte et les autres dans le second acte mais tous sur la scène au même moment ${ }^{2}$.

Le texte d'Agricola, De inventione dialectica (le titre est très parlant, De l'invention dialectique), est écrit en 1479, publié pour la première fois en 1515 grâce à un cercle de personnes qui se réunissent à Louvain, notamment autour d'Erasme de Rotterdam, qui fixe le texte dans sa version définitive. C'est un best-seller au XVI ${ }^{\text {ème }}$ siècle : plus de 70 éditions jusqu'en 1600. Un autre de ses grands succès, les Aphthonii Sophistae Progymnasmata serviront énormément à tous les poètes des XVI ème et XVII ${ }^{\text {ème }}$ siècles, ainsi qu'à tous les élèves dans les petites classes de rhétorique et de dialectique (avec la grammaire, ces disciplines forment le trivium).

Il revient à Pierre de la Ramée, au milieu du siècle, de continuer la réforme de la dialectique en retravaillant la partie jugement qu'Agricola n'avait pas mise en avant. Ce professeur de mathématiques au collège de Presles, puis professeur de philosophie et d'éloquence et de mathématiques au Collège royal a eu une grande influence sur l'enseignement de la rhétorique et de la dialectique, notamment dans l'Europe du Nord réformée ${ }^{3}$.

Si ces développements théoriques sont enseignés dans des écoles (celles dans lesquelles la langue est le latin), elles ont également des échos dans le milieu marchand des Pays-Bas de la seconde moitié du $\mathrm{XVI}^{\mathrm{ème}}$ siècle via les chambres de rhétorique, les rederijkerkamers, cette fois ci en langue vernaculaire (dialectes flamand, hollandais, brabant...). Une historiographie renouvelée 4 a montré comment ces chambres de rhétorique, formées sur le moule des guildes et des corporations, composées de marchands cultivés, ont joué un grand rôle dans la diffusion des savoirs (de la diffusion des idées de la Réforme jusqu'à l'initiation de marchands à la rhétorique par exemple) à travers l'organisation de concours de poésie ou de théâtre lors des « rituels urbains » comme les entrées princières ou les fêtes religieuses.

On voit ainsi se développer dans la seconde partie du seizième siècle une littérature sur la rhétorique en langue vernaculaire (couchée sur le papier et publiée, ce qui ne va pas de soi pour un rhétoriqueur de la première moitié du siècle $!^{5}$ ). Ce n'est ainsi que dans les années 1550 que sont publiées les premières rhétoriques néerlandophones, et dans les années 1580 pour les dialectiques néerlandophones ${ }^{6}$. Les chambres de rhétorique enseignent alors à leurs membres les bases du trivium, et agissent comme des scholen van welsprekendheid, des écoles du bien-parler. La dialectique tient à la fois le rôle dans ce contexte de discipline permettant la recherche de la vérité par l'argumentation et la persuasion et d'art de l'écriture de cette vérité dans un langage approprié ${ }^{7}$. Nous allons voir que l'algèbre prend un statut analogue dans l'évolution des arithmétiques.

\footnotetext{
2 Voir l'étude en histoire de la littérature de Marijke Spies sur les liens entre nouvelle rhétorique et poésie et théâtre (Spies, Op de questie, 1990) et le recueil d'articles de la même autrice (Spies, Rhetoric, 1999).

3 Sur les travaux rhétoriques de Pierre de la Ramée, voir (Ong, 1983).

4 Je renvoie pour les chambres de rhétorique notamment aux travaux menés par Anne-Laure van Bruaene : (van Bruaene, Brotherhood, 2005) et (van Bruaene, Guilds, 2006). Elle a par ailleurs réalisé une recension de ces chambres avec Arjel van Dixhoorn. Cette historiographie inscrit ces petites associations dans un contexte plus large de transmission des savoirs (chez Emily Peters), de leur institutionnalisation (chez Samuel Mareel) dans un pays qui s'est largement urbanisé.

${ }^{5}$ En 1555, l'imprimeur amstellodamois Jan Cauweel, en préface du Const van Rhetoriken de Matthijs de Castelein, explique que les chambres de rhétorique (lieux où l'oral a une grande importance) ne devraient pas avoir peur de confier les poèmes composés en leur sein à l'imprimé, pour laisser des traces aux générations futures de la langue néerlandaise. On a effet très peu de textes imprimés de la première moitié du siècle provenant d'auteurs liés aux chambres de rhétorique, l'oralité étant perçue alors comme garante de l'auteurité.

${ }^{6}$ En 1553, la Rhetorica de Jan van Mussem (c'est une œuvre inspirée de la Rhétorique ad Herennium, du De inventione de Cicéron, des Institutio oratoriae de Quintilien avec des morceaux du De conscribendis epistolis d'Erasme). L'art de la seconde rhétorique (codification de la poésie) est pour tous les auteurs jusque là de loin le plus important. Ce n'est cependant qu'en 1555 qu'apparait le premier traité contenant de la première rhétorique (ornementation des discours) en néerlandais : Matthijs de Castelein, Const van rhetoriken (six éditions jusqu'en 1616). Il est d'inspiration française (notamment des poètes de la Pléiade). Pour la dialectique, deux œuvres sont publiées en 1585, celle de l'universitaire Stevin dont on parlera plus loin et celle du facteur de chambre de rhétorique Hendrik Laurenszoon Spiegel, le Ruygh-bewerp vande Redenkaveling (abrégé de dialectique).

${ }^{7}$ La vérité comme le langage y étaient souvent considérés comme venant de l'Esprit Saint.
} 


\subsection{L'algèbre au carrefour de deux traditions mathématiques}

Les mathématiques et leur enseignement ont connu à bien des égards une évolution parallèle. En effet, ces mêmes marchands ont besoin d'une formation en arithmétique pour leurs calculs, on trouve au début du seizième siècle, surtout dans le sud des Pays-Bas où le commerce est florissant (Anvers et Bruges notamment), un grand nombre d'écoles en français et en dialectes néerlandais qui forment à tous niveaux au calcul, aux différentes règles de trois et à l'algèbre (rekenmeesters, franse scholen, etc. $)^{8}$. Cette arithmétique diffère alors au début du seizième siècle de l'arithmétique du quadrivium enseignée à l'université, c'est-à-dire dans notre cas à Louvain, qui s'en tient à une lecture assez littérale des Eléments d'Euclide.

Sous l'impulsion de savants du milieu du siècle, ces traditions se croisent à l'université : à Louvain, Gemma Frisius écrit en 1540 son Arithmeticae Practicae Methodus Facilis tandis que Jacques Peletier du Mans ou Pierre de La Ramée publient à Paris pour leur enseignement des manuels d'arithmétique écrit dans un français qui se veut populaire ${ }^{9}$. Dans les deux cas, c'est une arithmétique comme préparation à l'algèbre qui est voulue pour le plus grand nombre.

Au-delà de la convergence des traditions mathématiques marchandes et universitaires, c'est en effet la position de l'algèbre qui devient prépondérante dans l'architecture des savoirs : à la fois une méthode générale pour résoudre toujours plus de problèmes, elle permet l'unification de différentes règles de trois qui auparavant n'étaient applicables que dans un champ restreint (règle de compagnie, règle de société...), et est donc l'aboutissement d'un enseignement qui commence par les cas particuliers et qui ne passe véritablement du statut d'art à celui de science qu'avec l'évocation dans le dernier tiers du siècle des Arithmétiques de Diophante.

\subsection{Les convergences entre dialectique et algèbre}

On observe donc des convergences entre la dialectique et l'algèbre : historiques par rapport à leur enseignement et leur diffusion dans des milieux différents (marchands et universitaires) qui finissent par s'entremêler au milieu du siècle, épistémologiques pour leur rôle dans l'organisation et l'unification des savoirs.

L'évolution historique des traditions arithmétiques montre comme on l'a vu une unification par l'algèbre et parallèlement, la dialectique était selon Pierre d'Espagne, au XIII ${ }^{\text {ème }}$ siècle « l'art des arts et la science des sciences ». Les deux disciplines revendiquent ce statut au milieu du XVI ${ }^{\text {ème }}$ siècle et l'on peut donc problématiser leurs liens éventuels.

Ainsi que le fait Giovanna Cifoletti de manière programmatique dans (Cifoletti, Introduction, 2006) et dans le cas français dans (Cifoletti, Peletier, 1992), il s'agit de montrer que dans certains milieux où algèbre et rhétorique/dialectique sont utilisées, les deux disciplines ont une influence l'une sur l'autre. Ce papier s'inscrit dans cette démarche en montrant par différentes approches, dont celle par les séries de problèmes, comment Simon Stevin, dans la seconde moitié du XVI ème siècle, utilise sa dialectique pour écrire son arithmétique.

\section{Simon Stevin}

\subsection{Eléments biographiques}

Stevin est né dans une famille marchande en 1548 à Bruges ${ }^{10}$. Après quelques fonctions dans les villes de Bruges et d'Anvers comme marchand et banquier, il s'en va en Hollande, à Leyde, en

\footnotetext{
${ }^{8}$ Lire sur les arithmétiques néerlandophones marchandes et universitaires l'excellent livre de Marjolein Kool (Kool 1999).

${ }^{9}$ Lire les contributions dans ce volume de Christelle Serra et de Giovanna Cifoletti.

${ }^{10}$ Les éléments biographiques sont tirés des travaux de E.J. Dijksterhuis et d'un ouvrage plus récent : (Dijksterhuis, 1943) et (Devreese \& Vanden Berghe, 2008).
} 
1581, à une époque où le sud est pris dans une guerre d'indépendance économico-religieuse (guerre de Quatre-vingts ans, 1568-1648). Il s'inscrit à l'université de la ville entre 1583 et 1590, dans le cursus des arts, en même temps qu'il s'adonne à l'écriture de nombreux textes théoriques et pratiques en mathématiques et en mécanique, mais aussi en dialectique, en grammaire ou en politique. Stevin devient ingénieur militaire pour Maurice de Nassau, comte d'Orange, à qui il enseigne les différentes disciplines mathématisées, comme l'astronomie, la navigation ou la musique. Il publie des œuvres militaires (un traité de fortifications, un autre pour la gestion des camps militaires) peu après 1600, et se consacre pleinement à son activité de quartier-maître général pendant la guerre de Trente ans. Il meurt en 1620.

En observant ses œuvres complètes, nous constatons que Simon Stevin écrit et publie en fonction de son actualité immédiate et des domaines très variés qu'il a à connaître : un traité de géométrie quand il est professeur de collège, un traité de politique quand l'indépendance des Pays-Bas demande la recherche d'un dirigeant, des traités militaires quand il est dans l'armée de Maurice de Nassau etc... Stevin dispose d'une méthode qui lui permet de « réduire en art ${ }^{11}$ des pratiques venant d'horizons très divers, de codifier par le même « art de l'écriture »et grâce au même «style mathématique » ${ }^{12}$ des théories portant sur des thématiques aussi variées que possible. Dialectique et mathématiques semblent donc bien avoir cette potentialité d'unifier des savoirs.

\subsection{Statuts de la dialectique et de l'algèbre chez Stevin}

Démontrer que mathématiques et dialectique sont liées chez Simon Stevin demande de lire en parallèle deux textes publiés en 1585 chez Christophe Plantin à l'université de Leyde : L'Arithmetique ${ }^{13}$ et Dialectike ofte bewijsconst (titre que l'on peut traduire par Dialectique ou art de la démonstration ${ }^{14}$ ). Dans le premier, il décrit l'algèbre comme «l'inexhauste fontaine d'infiniz théorèmes arithmetiques, revelatrice des mysteres cachez en nombres ${ }^{15}$ tandis qu'il décrit dans le second la dialectique comme « apprenant à juger du vrai et du faux et ouvrant la voie aux plus profonds mystères de la nature ${ }^{16}$. Le savant flamand nous explique qu'il ne peut écrire de mathématiques en langue flamande sans avoir préalablement écrit une dialectique dans cette même langue :

«En premier lieu, après que certains amis et compatriotes de ma fréquentation, ignorant les langues étrangères mais tout de même amateurs éminents des arts, aient compris que nous ferions imprimer quelque chose, en langue étrangère il est vrai, en mathématiques, ils m'ont non seulement prié de faire une chose pareille en notre propre langue (comment peut-on refuser à ceux qui le désireraient ?), mais ont même voulu me persuader qu'il était du devoir de chacun, pour sa patrie, de se soucier du devenir de sa fortune et de la préférer à celle de ses amis et de ses parents, voire même de la sienne. [...] Mais voyant qu'il se trouve un style dialectique [enartsmathématiques], c'est-à-dire des tournures et des mots, que l'on appelle Vocabula Artium, lesquels, lorsqu'ils sont utilisés à la latine (comme le font ceux qui s'en servent en néerlandais), ne sont pas bien compris de ceux qui ne connaissent pas bien la chose ; voyant aussi qu'en néerlandais leur signification reste inconnue, étant donné que jusqu'ici il

\footnotetext{
11 Voir (Dubourg Glatigny \& Vérin, 2008).

12 Les deux dernières expressions sont de Stevin lui-même, respectivement dans (Stevin, Dialectike, 1585) et dans (Stevin, Wiscondige, 1608).

13 Voir (Stevin, L'Arithmetique, 1585).

14 En néerlandais actuel, bewijs signifie un certificat, le dictionnaire franco-latino-flamand de Plantin (1573) traduit bewijs par connaissance.

15 (Stevin, L'Arithmetique, 1585), p.398.

16 Sous-titre de (Stevin, Dialectike, 1585), « Leerende van allen saecken recht ende constelick oirdeelen; oock openende den wech tot de alderdiepste verborghentheden der natueren. »
} 
n'existait pas de dialectique en néerlandais, je me trouvais quasi forcé, par conséquent, de faire cette dialectique néerlandaise. » 17

Au-delà du "patriotisme" linguistique qu'on retrouverait chez un Du Bellay en France et qui est un topos des rhétoriqueurs néerlandais en particulier depuis le début de la guerre d'indépendance des Provinces-Unies, le thème a une grande importance sur le plan de la logique. C'est ce constat que fait Stevin de l'indissolubilité de la logique (Sect. 3) et de la langue utilisée (Sect. 4) qui l'oblige à écrire un traité de dialectique.

\subsection{La méthode dialectique}

Deux points de cette œuvre vont particulièrement nous intéresser ici. D'une part, Stevin remplace l'invention et la disposition cicéroniennes par les notions de définitions et opérations : les définitions étant naturellement aux lettres ce que les opérations sont aux mots, les premières doivent être la matière avec laquelle on fabrique les secondes :

«La nature nous apprend donc que les définitions des noms propres [aux concepts] de la dialectique sont la première des choses à connaître, et que par chacun de leur potentiel ordonnancement il y a autant de manière équivalente de les étendre. Car de la même façon que les lettres sont dans la grammaire, de la même façon les noms [desconcepts] comme Matière, Forme, Genre, Espèce, etc... sont dans la dialectique.

Les définitions forment donc naturellement et convenablement la première partie de la dialectique et de tous les autres arts, avec lesquelles les opérations qui les suivent sont faites et sont connues. ${ }^{18}$

Ces termes définissent, on le verra plus loin, les deux grandes parties de toutes les œuvres de Stevin et sont particulièrement structurants pour des textes destinés à être imprimés et étudiés sans professeur. D'autre part, l'auteur flamand propose une méthode en quatre points pour organiser les savoirs d'une discipline :

«En premier lieu, il est nécessaire pour bien distribuer, de connaitre les parties, ou espèces, des ensembles donnés, ou genres, sans lesquels la science, ne peut se justifier de ses particularités.

En second lieu, on partage selon la dichotomie, toujours en divisant en deux, mais si l'on rencontre des passages où l'on ne peut le faire convenablement, on divisera en le moins possible selon ce que l'opportunité exige, en trois, en quatre, \&c.

En troisième lieu, que les parties, ou espèces, réciproques (contraires ou autres) soient mises au même niveau, quand on peut les trouver. Car qui voudrait différencier les animaux, en disant d'aucun être soit cheval, soit jument, ferait une division maladroite; vu que toute jument est un cheval.

En quatrième lieu, que les parties, ou espèces, pris en tant qu'ensembles, mesurent les effets ou les puissances (de la même façon qu'en l'être humain il vient deux espèces que sont l'homme et la femme), comme les espèces de l'homme sont Jean, Pierre, Jacques, etc., et ceci avec chaque mot, etc. ou quelque chose semblablement en puissance, nous entendons tous les autres qui nous sont imprenables dans leur singularité, car tant que ce n'est pas inséparable, autant il faut aller plus loin dans la partition. ${ }^{19}$

Ce à quoi l'on aboutit par cette méthode est un arbre formé d'accolades. On obtient ainsi des ramifications de concepts, des mots qui sont distribués, étendus sur une page : ce sont les tables

\footnotetext{
${ }^{17}$ Préface de la Dialectike ofte bewijsconst, $\mathrm{f} 3 \mathrm{v}-\mathrm{f} 4 \mathrm{v}$, la traduction de ce passage est de Theo Verbeek dans le seul article que je connaisse dédié uniquement à ce texte - délaissé par l'histoire des sciences dont Stevin est pourtant un lieu commun, délaissé également de manière compréhensible par l'histoire de la dialectique. (Verbeek, 2005).

18 (Stevin, Dialectike, 1585), dans l'appendice pp.107-108. La citation continue sur le fait que ceux qui utilisent invention et ordre appartiennent au passé, tandis que ceux qui aimeront à mettre par écrit leur opinion le feront convenablement grâce à la division en définition et opérations.

19 Dialectike ofte bewijsconst, p. 57, ma traduction. (Stevin, Dialectike, 1585). Les quatre points de la méthode que propose Stevin sont à placer chronologiquement entre les trois points de celle de Pierre de La Ramée (que Stevin connaît peut-être par son passage à la chambre de rhétorique de Bruges, par les traductions qui circulent pour les collèges ou les enseignements des professeurs Snell père et fils à l'université de Leyde) et les quatre points de celle du Discours de la méthode de Descartes (Descartes connaît peut-être la dialectique de Stevin par la republication qui en a été faite en 1621 à Rotterdam, peut-être via Beeckman ou Snell ?).
} 
dichotomiques (voir des exemples plus loin), mises au goût du jour par Rudolph Agricola et diffusées très largement par l'imprimerie dans les travaux de Pierre de la Ramée et de ses disciples à la fin du siècle. D'une part, il est important que ces tables soient bien faites car elles participent à la fois de l'invention et de la disposition, d'autre part, elles permettent l'exploration (subjective, suivant l'ordonnancement des mots dans les accolades) et l'épuisement des possibles évoqués dans le quatrième point de la méthode.

\subsection{Mathématiques et dialectique dans le flamand de Stevin}

Si le latin est une langue par cas et déclinaison (par exemple un cas pour le complément de nom), le flamand est une langue qui, comme l'allemand, peut fabriquer des mots en les juxtaposant sans limite. Or la langue doit pour les continuateurs de Pierre de La Ramée refléter la pensée, qui elle-même est à l'image du monde. Pour Simon Stevin, puisque la pensée est rapide quand elle considère des réalités simples et plus lente lorsqu'elle considère des réalités complexes, les réalités simples doivent être dénotées par des mots monosyllabiques et les réalités complexes par des mots plus longs. Encore une fois, le contenu, par exemple mathématique, ne pourra pas être séparé de la langue utilisée et de la manière d'articuler les concepts fournie par la dialectique. ${ }^{20}$

La volonté qu'a Stevin de montrer que le flamand est la langue scientifique par excellence le pousse à la comparer au latin et au grec. Il y a, nous dit-il, listes à l'appui, 742 verbes et 1428 noms communs monosyllabiques en flamand, contre 5 et 158 en latin, et 0 et 220 en grec. Le monosyllabisme associé à la composition des mots par concaténation fait du flamand une langue plus adaptée que les autres à décrire la réalité du monde.

La vision de la langue rejoint chez lui la manière de travailler en grammaire et en géométrie. Dans l'une, on part des lettres pour former des mots, dans l'autre, les concepts définis en premier sont les plus généraux et les plus simples, et au fur et à mesure des démonstrations, on s'achemine vers des objets plus particuliers mais aussi plus complexes. Le monde est ainsi mathématisé par l'intermédiaire de la langue, grâce à ce parallélisme entre la longueur des mots et la complexité du monde ${ }^{21}$.

Parce qu'il est souvent le premier à aborder un sujet en néerlandais, Stevin a créé un langage (quelques centaines de néologismes) selon ses besoins et en accord avec sa pensée ${ }^{22}$. Il est ainsi l'un des contributeurs les plus importants de la langue néerlandaise en matière de sciences et de techniques. Parmi ces néologismes, on observe que bewijsconst pour dialectique et wiskunde pour mathématiques sont clairement apparentés (respectivement en dialectes flamand et hollandais).

En 1608, Stevin complète la méthode décrite plus haut en y adjoignant l'anaphore, cette figure de style rhétorique qu'il réemploie pour désigner le fait de nommer des concepts proches par des expressions proches.

Malgré le fait que L'Arithmetique soit en français et non en flamand (l'éditeur, Christophe Plantin, aura-t-il eu peur de ne pas vendre suffisamment de tels livres s'il était écrit en flamand ?), on pourra y trouver des considérations sur le langage. On remarque par exemple un cas de l'anaphore mentionnée plus haut : Stevin se refuse d'utiliser le mot équation ${ }^{23}$ et lui substitue l'expression « quatriesme proportionel algebraique » :

\footnotetext{
${ }^{20}$ Pour cette articulation entre la langue utilisée, les contenus scientifiques et la rhétorique/dialectique, voir l'article (Cifoletti 1999) qui traite de «l'humanisme vulgaire » dans le cas français : « De même que le poète doit avant tout s'exprimer dans sa langue maternelle, le scientifique devra utiliser sa propre langue vulgaire, pour la transformer en une langue scientifique : il s'agit là de thèses que Jacques Peletier du Mans développe surtout dans ses ouvrages d'arithmétique et d'algèbre. [...] Du point de vue des acteurs, au moins, l'enjeu est le statut du discours par rapport à la vérité, autrement dit le style de la science. » (Sect. 21).

21 Voir (Stevin, Wiscondige, 1608) et (Van der Wal, 1993). L'idée des mots monosyllabiques plus nombreux en néerlandais que dans les autres langues se trouve également chez (Spiegel, 1585).

22 Voir (Devreese \& Vanden Berghe, 2008), chapitre 8, pp. 201-212.

23 Le mot équation étant très rare à l'époque dans la langue française, on peut déduire que Stevin le reprend chez Jacques Peletier du Mans. Ces deux auteurs ont comme on l'a vu les mêmes types de réflexions sur le langage.
} 
« Nous le nommons ainsi, parce qu'il est plus commode à la doctrine; Car puis qu'il y a touiours donnez trois termes, ausquels on cherche vn quatriesme proportionel (comme apparoistra en son lieu) pourquoi ne s'appelleroit ceci pas aussi bien inuention de quatriesme proportionel, comme en tous autres? [...] Car ce mot d'equation à faict penser aux apprentifs, que c'estoit quelque matiere singuliere, laquelle toutesfois est commune en la vulgaire arithmetique, car nous cherchons à trois termes donnez, vn quatriesme proportionel. » (p. 264).

De même, la règle d'algèbre est considérée (et renommée) comme la règle de faux des nombres algébriques (p. 398).

\subsection{Les œuvres de Stevin comme séries de problèmes}

Toutes les œuvres de Simon Stevin sont écrites sous la forme de séries de définitions et de séries de problèmes, précédées par un diagramme dichotomique. On peut donc définir la sérialité dans les œuvres de Stevin par l'appartenance à une unité textuelle issue d'un même diagramme dichotomique, cette sérialité ne séparant jamais définitions et opérations. La dichotomie est par suite l'élément clé de la construction d'une série (de problèmes) chez Stevin puisque la table dichotomique joue le rôle de tables des matières du texte : elles sont affichées au début de toutes ses œuvres, et le texte suit l'ordre des ramifications de l'arbre.

Dans son texte de dialectique, Stevin nous propose, à titre d'exemple de la méthode expliquée plus haut, de reconstruire un texte qu'il avait publié deux ans plus tôt sur les tables d'intérêts. Les Tafelen van Intrest sont traduites en français par ses soins dans la Pratique d'Arithmetique.

La première des choses à faire est de séparer les définitions des opérations, on obtient donc ceci :

$$
\text { La règle d'intérêt a deux parties }\left\{\begin{array}{l}
\text { Définitions } \\
\text { Opérations }
\end{array}\right.
$$

Ensuite, la méthode proprement dite s'applique, en commençant par trouver les concepts qui seront définis puis disposés selon les trois derniers lieux, ici, ceux de capital, d'intérêt, de taux d'intérêt, d'intérêts simples, composés, profitables et dommageables. Les regroupements seront les suivants : on opposera intérêts simples à intérêts composés, et les profitables aux dommageables. Une ambiguïté surgit quant à la hiérarchie des genres et des espèces, puisqu'un intérêt simple peut être ou profitable ou dommageable, de la même façon qu'un intérêt profitable peut être ou simple ou composé. Stevin explique que l'on peut alors choisir mais qu'il semble plus correct de placer les simples et composés avant les profitables et dommageables, les premières étant des notions plus fondamentales que les secondes. D'où la table suivante :

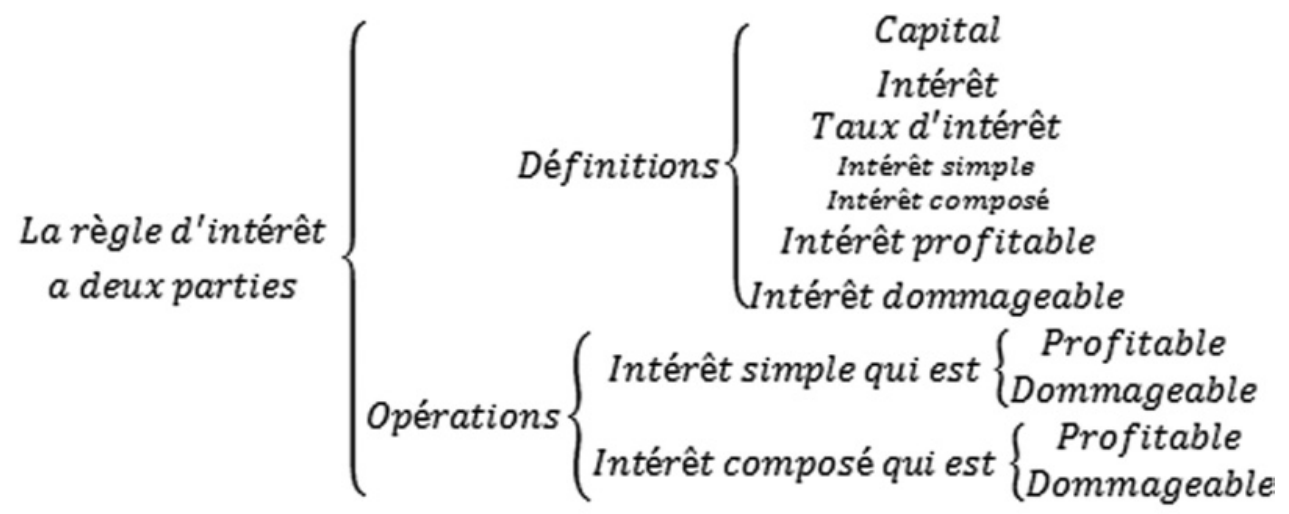


La série de problèmes obtenue prend donc l'ordre suivant (en reprenant la traduction française de la Pratique d'Arithmetique) que je résume dans le tableau suivant (avec les numéros des définitions et des opérations en deuxième colonne, la définition dans son entièreté ou les énoncés seuls des problèmes dans la troisième colonne) :

\begin{tabular}{|c|c|c|}
\hline \multirow[t]{7}{*}{ Définitions } & I. & Capital est la somme, de laquelle on compte l'Interest. \\
\hline & II. & $\begin{array}{l}\text { Interest est la somme que l'on compte de l'arrierage du capital } \\
\text { pour quelque temps. }\end{array}$ \\
\hline & III. & $\begin{array}{l}\text { La Raison qu'il y a de l'interest au capital, nous l'appelons Raison } \\
\text { d'interest. }\end{array}$ \\
\hline & IIII. & $\begin{array}{l}\text { Interest simple est celui, que l'on compte seulement } \\
\text { du capital. }\end{array}$ \\
\hline & $\mathrm{V}$. & $\begin{array}{l}\text { Interest composé est celui, que l'on compte du capital ensemble } \\
\text { de l'arrierage. }\end{array}$ \\
\hline & VI. & Interest prouffitable est celui, qu'on aiouste au capital. \\
\hline & VII. & Interest dommageable est celui, qu'on soubstraict du capital \\
\hline \multirow[t]{4}{*}{ Opérations (en propositions) } & I. & $\begin{array}{l}\text { Estant declaré capital temps \& raison d'interest simple \& } \\
\text { prouffitable : Trouuer l'interest. }\end{array}$ \\
\hline & II. & $\begin{array}{l}\text { Estant declaré capital, temps, \& raison d'interest simple \& } \\
\text { dommageable : Trouuer leur valeur. }\end{array}$ \\
\hline & III. & $\begin{array}{l}\text { Estant declaré capital, temps, \& raison d'interest composé \& } \\
\text { prouffitable : Trouuer combien monte } \\
\text { le capital avec son interest. }\end{array}$ \\
\hline & IIII. & $\begin{array}{l}\text { Estant declaré capital, temps, \& raison d'interest composé } \\
\text { dommageable : Trouuer leur valeur. }\end{array}$ \\
\hline
\end{tabular}

On voit donc la correspondance qui existe entre l'ordre des «feuilles » dans les ramifications de la table dichotomique d'une part, et l'ordre de la série de définitions et de la série de problèmes (ici nommés propositions) d'autre part. De plus, la table dichotomique peut être comprise comme une table des matières du texte, ce qui explique sa position initiale dans les œuvres de Simon Stevin.

Voir comment la méthode décrite est utilisée dans l'Arithmetique est plus compliqué, car c'est un texte beaucoup plus long, qui comporte 103 définitions et 81 problèmes. Par ailleurs, l'organisation du traité d'arithmétique est somme toute assez classique et habituelle, et semble procéder par difficulté croissante autour des quatre opérations : d'abord les nombres entiers, ensuite les fractions... pour en arriver finalement aux nombres algébriques, et donc à la résolution des équations, ce qui ouvre la voie à une traduction des quatre premiers livres de l'Arithmetique de Diophante.

Il me semble cependant intéressant d'aller chercher l'archéologie de la construction d'une telle série, car aussi banale soit-elle, elle n'en reste pas moins ancrée dans un contexte très particulier, en exhumant ce qu'elle contient d'art de l'écriture dialectique. Le diagramme dichotomique nous donne de bons indices : l'opposition définitions et opérations, le nombre est opposé à ses effets (mot qu'il faut comprendre comme la réciproque de la cause - terme expliqué en détail dans la dialectique), les oppositions sont hiérarchisées et correspondent ensuite dans le texte à des parties ou des « distinctions », à des définitions et des problèmes. Epuiser le concept (le quatrième point de la méthode) consiste ici à le décliner selon des opérations élémentaires. Comparant l'ordre des problèmes avec ce que l'on voit sur le diagramme, on comprend l'artifice graphique utilisé en bas à droite pour éviter d'avoir à répéter six fois le même sous-diagramme. On remarque également que les 81 problèmes ne figurent pas comme 


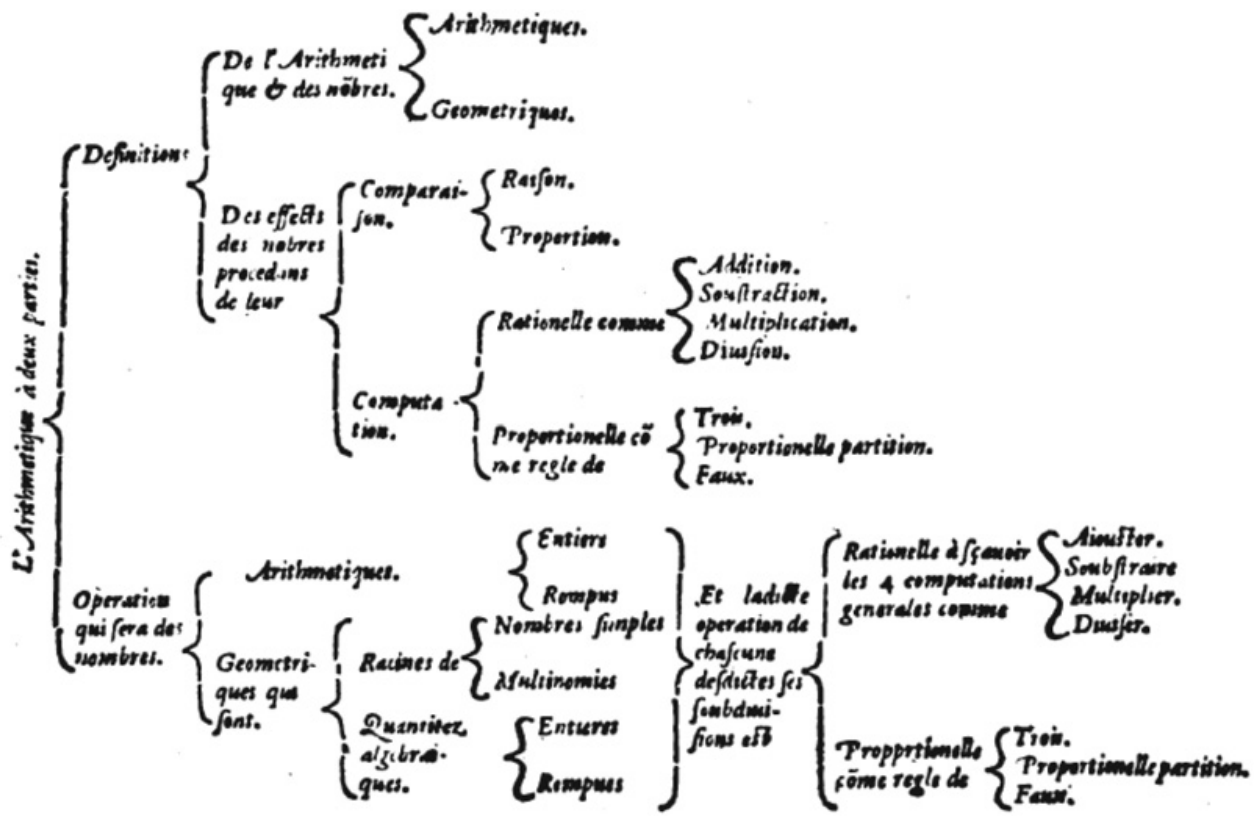

Figure 1. Table dichotomique de L'Arithmetique (1585), f9v.

« feuilles » de l'arbre dichotomique (il n'y en aurait que 42 si l'on suivait l'arbre - six types de nombres pour sept opérations différentes).

Ces absences laisseraient penser que ce ne sont pas autour de sept opérations mais seulement de quatre qu'est construit le texte. En effet, seuls les problèmes concernant les quatre premières opérations sur les types de nombres sont effectivement traités, les autres permettraient a priori de passer d'un niveau de difficulté un autre (des nombres entiers aux nombres fractionnaires par exemple). Pour mieux le visualiser, je me suis servi d'un tableau modélisant la série de problèmes numérotés de 1 à 81 (qui constitue la seconde partie du livre).

D'autres modes de représentation peuvent être utilisées pour modéliser une série de problèmes : des bulles reliées avec des flèches, des codes de couleurs etc... Le tableau a cet avantage de comporter des vides qu'il faut pouvoir expliquer. Il permet donc d'aller au-delà du texte. Cette modélisation n'est pas parfaite, elle laisse des choses de côté et en montre d'autres. Cependant, on peut se demander si nous ne surinterprétons pas le mode de pensée de l'auteur (ici Stevin) en plaquant sur son texte une telle schématisation bidimensionnelle ? Les savants à la suite de Pierre de la Ramée ont une pensée classificatoire très forte (en grammaire comme en sciences) : il s'agit de trier les savoirs dans des catégories qui ne se recoupent pas, d'où l'importance accordée aux oppositions strictes entre paires de concepts et par suite à la dichotomie et les tables correspondantes. Ces dernières sont comme le tableau utilisé plus haut, à deux dimensions (qui correspondent aux deux dimensions de la page imprimée), bien que ce qui est dévoilé soit de nature différente. La table dichotomique utilise deux échelles : du haut vers le bas pour un ordre de la simplicité vers la complexité, de la gauche vers la droite pour un ordre allant du général au particulier. Le tableau quant à lui permet de clarifier les notions répétitives (ici les quatre opérations) mais ne distingue pas les passages entre des sous-séries de problèmes.

A partir de ce tableau, on peut se poser plusieurs questions et comprendre un peu mieux l'ordre de la série de problèmes mais également les vides du tableau. Par exemple, l'entremêlement du traitement des postposées quantités (expressions algébriques avec plusieurs inconnues) et de celui des équations des degrés un à quatre est aussi à questionner. De même, on peut se demander pourquoi les problèmes 44 à 
Les séries de problèmes, un genre au carrefour des cultures

\begin{tabular}{|c|c|c|c|c|c|c|c|c|c|}
\hline & \multicolumn{2}{|c|}{$\begin{array}{c}\text { Nombres } \\
\text { arithmétiques }\end{array}$} & \multirow[t]{2}{*}{$\begin{array}{l}\text { Nombres } \\
\text { radicaux }\end{array}$} & \multicolumn{3}{|c|}{ Multinomies radicaux } & \multicolumn{3}{|c|}{ Nombres algébriques } \\
\hline & Entiers & Rompus & & Entiers & Rompus & Racines & Entiers & Rompus & Postposées \\
\hline Addition & 1 & 10 & 24 & 28 & 32 & 42 & 51 & 59 & 64 \\
\hline Soustraction & 2 & 11 & 25 & 29 & 33 & 43 & 52 & 60 & 65 \\
\hline Multiplication & 3 & 12 & 22 & 26 & 30 & 40 & 49 & 57 & 62 \\
\hline Division & 4 & 13 & 23 & 27 & 31 & 41 & 50 & 58 & 63 \\
\hline $4^{\mathrm{e}}$ proportionnel & \multicolumn{2}{|c|}{14} & \multicolumn{4}{|c|}{44} & \multicolumn{2}{|c|}{$\begin{array}{c}66 \text { à } 78 \\
\text { (équations) }\end{array}$} & 79 et 80 \\
\hline Moyen prop. & & & \multicolumn{4}{|c|}{45} & & & \\
\hline Prop. partition & \multicolumn{2}{|c|}{15} & \multicolumn{4}{|c|}{46} & & & \\
\hline Fausse position & \multicolumn{2}{|c|}{16} & \multicolumn{4}{|c|}{47} & \multicolumn{2}{|c|}{81} & \\
\hline Dble fausse pos. & \multicolumn{2}{|c|}{17} & \multicolumn{4}{|c|}{48} & & & \\
\hline PGCD & 5 & & & & & & 53 & & \\
\hline Premier rompu & & 6 & & & & & & 54 & \\
\hline$a+b / c--d / c$ & & 7 & & & & & & 55 & \\
\hline $\mathrm{d} / \mathrm{c}---\mathrm{a}+\mathrm{b} / \mathrm{c}$ & & 8 & & & & & & & \\
\hline $\begin{array}{l}\text { Réduction } \\
\text { dénominateur }\end{array}$ & & 9 & & & & & & 56 & \\
\hline Racine & & & 18 & & 39 & & & 61 & \\
\hline Réd. Racine & & & 19 & & & & & & \\
\hline Commensurabilité & & & 20 & & & & & & \\
\hline Raison & & & 21 & & 34 & & & & \\
\hline Binomies & & & & & 35 à 38 & & & & \\
\hline
\end{tabular}

48 viennent si tard, après seulement le traitement des multinomies radicaux (expressions mathématiques prenant la forme d'une somme ou d'un rapport de somme de plusieurs nombres radicaux). C'est en faisant ce genre de tableau que l'on voit également ce qui n'est pas fait. Ainsi, ces problèmes 44 à 48 doivent servir à mon sens également aux colonnes vides correspondantes aux multinomies radicaux, les techniques mathématiques mises en œuvre s'y ramenant. Ces problématiques sont au croisement de ce qu'impose la forme sur le fond et le fond sur la forme, étant donné qu'un texte continu ou présenté sous forme de dialogue aurait pu s'affranchir de la table dichotomique, et être sans doute plus précis sur le traitement réservé aux multinomies radicaux par exemple.

De plus, la traduction des quatre premiers livres des Arithmétiques de Diophante en français doit être incluse dans le vertigineux $81^{\mathrm{e}}$ problème intitulé assez laconiquement : «Estant proposé question qui se solue par Algebre : la soluer par Algebre ». Ces livres contiennent de nombreuses questions (c'est le mot employé pour exemplifier la résolution d'une partie de problème et dont l'énoncé est entièrement instancié) qui permettent de compléter les vides du tableau. 
Ces questions une fois posées, nous pouvons voir que l'ossature de la série de problèmes est bien celle fournie par la table dichotomique, avec ses sept opérations.

La fin de la série de problèmes et la traduction de Diophante marque le point d'arrivée d'un lecteur que Stevin aura conduit marche après marche depuis le calcul d'une somme de deux nombres entiers jusqu'au « seuil des mathématiciens les plus grands ${ }^{24}$. La suite de l'œuvre, La Pratique d'Arithmetique, mais également d'autres traités plus tardifs, chercheront à décliner la méthode générale algébrique dans des cas particuliers : la notation de l'inconnue (1), de son carré (2), de son cube (3), etc... signifiant tour un tour un dixième, un centième, un millième... dans La Disme (texte destiné aux marchands, aux fabricants de tonneaux...), un degré, une minute, une seconde...dans un texte préparatif à l'astronomie, etc..

\section{Conclusion}

Simon Stevin s'est approprié à la fin du XVI ème siècle deux héritages. Celui issu de la réforme de la dialectique et de la rhétorique, à la suite de Rudolph Agricola, d'Erasme et de Pierre de La Ramée, lui a donné un cadre de pensée logique et linguistique. L'autre, issu des recompositions qui ont eu cours au milieu du siècle en arithmétique chez Gemma Frisius, Jacques Peletier du Mans ou Guillaume Gosselin, est révélateur de la position centrale accordé à l'algèbre (chez Stevin par le recours aux notations algébriques dans des contextes très différents).

Peu après son entrée à l'université, Stevin publie en 1585 deux textes dont le public pourrait correspondre à celui des chambres de rhétorique qu'il connaît (la Heilige Gheest de Bruges ou De Eglentier en Amsterdam). On a ainsi montré que ces deux héritages se croisent et se manifestent conjointement dans L'Arithmetique et dans la Dialectike. Si Euclide reste une autorité, la présentation axiomatique est négligée et le lecteur est invité à relativiser la certitude du savoir exposé : si la table dichotomique représente un choix parmi d'autres de hiérarchisation et d'oppositions, un autre auteur aurait pu obtenir une mathématique bien différente avec une autre table, comme l'a montré l'exemple des tables d'intérêts.

L'importance qu'a prise l'imprimerie durant le siècle, et donc celle de l'écrit par rapport à l'oralité (qui pourrait être mise en scène dans une série de problèmes dialoguée), ainsi que la réforme de la dialectique, mais aussi bien d'autres évolutions, permettent à un individu de lire - éventuellement seul, comme le rappelle Stevin - un texte en ayant les codes qui lui permettent de comprendre l'argumentation de l'auteur absent, et donc d'entrer dans un débat virtuel avec lui. Les notations algébriques ou la construction de tables dichotomiques montrent bien l'importance du support matériel de la pensée dans la hiérarchie des disciplines et la transmission de leurs contenus.

Les problématiques mises en avant par la dialectique comme la méthode d'invention et de jugement, retravaillée pour obtenir un art de l'écriture unique, montrent le souci d'une place cruciale faite à la langue vernaculaire et à son utilisation pour des savoirs aussi bien théoriques que pratiques. Le parallèle entre algèbre et dialectique permet alors de comprendre L'Arithmetique comme un cheminement qui utilise l'ordre de la prudence (selon la terminologie ramusienne, c'est-à-dire en partant des notions les plus faciles pour terminer aux plus difficiles), à l'image du cursus suivi dans la formation rhétorique grâce aux progymnasmata, permettant à l'étudiant d'acquérir petit à petit des fondements algébriques réutilisables dans une large variété de problèmes. Il n'y a alors qu'un pas pour transformer définitivement l'art de l'écriture dialectique en un style mathématique, faisant de l'algèbre une méthode universelle pour les savoirs mathématisables.

24 (Stevin, Dialectike, 1585), f4v. 
Les séries de problèmes, un genre au carrefour des cultures

\section{Références}

Cifoletti, G. (1992). Mathematics and Rhetoric. Peletier and Gosselin and The Making of the French Algebraic Tradition. Princeton.

Cifoletti, G. (1999). L'idéal de la science. Enquête (7), pp. 283-303. Consultable en ligne http: //enquete.revues . org/1589 (consulté 29.10.15).

Cifoletti, G. (2006). Mathematics and rhetoric, introduction. Early Science and Medicine, 11(4), pp. 369-389.

Devreese, J., \& Vanden Berghe, G. (2008). "Magic is no magic”: The wonderful world of Simon Stevin. Southampton: WIT.

Dijksterhuis, E. J. (1943). Simon Stevin. 's Gravenhage: Martinus Nijhoff.

Dubourg Glatigny, P., \& Vérin, H. (2008). Réduire en art. La technologie de la Renaissance aux Lumières. Paris: Editions de la MSH.

Kool, M. (1999). Die conste vanden getale. Hilversum: Verloren.

Mack, P. (2011). A History of Renaissance Rhetoric 1380-1620. Oxford: Oxford University Press.

Ong, W. J. (1983). Ramus, method, and the decay of dialogue: from the art of discourse to the art of reason. Cambridge, Massachussets: Harvard University Press.

Spiegel, H.L. (1585). Ruygh-bewerp vande Rekenkaveling ofte Nederduytsch Dialectike. Leyde: Christophe Plantin.

Spies, M. (1990). “Op de questie...": Over de structuur van 16e-eeuwse zinnespelen. De nieuwe taalgids, 83(2), 139-150.

Spies, M. (1999). Rhetoric, Rhetoricians and Poets. Amsterdam: Amsterdam University Press.

Stevin, S. (1585). Dialectike ofte bewijsconst. Leyde: Christophe Plantin.

Stevin, S. (1585). L'Arithmetique et la Pratique d'Arithmetique. Leyde: Christophe Plantin.

Stevin, S. (1608). Wiscontige Gedachtenissen inhoudende t'ghene daer hem in gheoeffent heeft... Leyde: Jan Bouwensz.

van Bruaene, A.-L. (2005). Brotherhood and Sisterhood in the Chambers of Rhetoric in the Southern Low Countries. The Sixteenth-Century Journal, 36(1), 11-35.

van Bruaene, A.-L. (2006). "A wonderfull tryumfe, for a wynnyng of a pryse": Guilds, Ritual, Theater and the Urban Network in the Southern Low Countries, ca 1450-1650. Renaissance Quarterly, 59(2), 374-405.

Van der Wal, M.J. (1993). Logic, Linguistics, and Simon Stevin in the context of the $16^{\text {th }}$ and $17^{\text {th }}$ centuries. Dans K.R. Jankovsky, History of Linguistics (pp. 147-156). Amsterdam: John Benjamins Publishing Company.

Verbeek, T. (2005). La Dialectike (1585) de Simon Stevin. Dans C. Secretan, \& P. den Boer, Simon Stevin De la vie civile 1590 (pp. 181-190). Lyon : ENS éditions. 